\title{
The impact of COVID-19 in diabetic patient
}

\author{
Antonio Vitiello ${ }^{1}$ and Francesco Ferrara ${ }^{1}$ \\ ${ }^{1}$ Azienda Unità Sanitaria Locale Umbria 1
}

May 26, 2020

\begin{abstract}
Beckgroung: The global pandemic Sars-Cov-2 (COVID-19) is causing thousands of deaths worldwide, and is one of the greatest health challenges ever faced in human history. Sars-Cov-2 infection can cause fatal lung injuries caused by a generalized inflammatory state associated with multi-organ dysfunction. Objective: In this context, it is essential to recognize the factors that increase the risk of viral infection. People with pre-existing conditions, such as diabetes, are at greater risk of complications and death caused by COVID-19. Materials and Method: Old age, possible chronic drug therapies, kidney failure, hyperglycemia, heart disease, are predictive factors of a bad outcome for the diabetic patient. The regulation of glycaemia and the adoption of appropriate measures are critical aspects to be taken into consideration for the diabetic patient in this pandemic period, especially in the patient with ongoing infection. Results: In the latter, the use of drugs used to fight COVID-19 infection, such as antivirals or immunomodulants, must be well controlled to avoid possible drug interactions and major complications. People with diabetes fall into the category of fragile and at-risk population, and if a COVID-19 infection is also ongoing the patient must be managed optimally, trying to fight the virus but also without neglecting homeostasis and glycemic control. Conclusion: This analysis highlights current knowledge and challenges for the prevention and management of patients with diabetes and COVID-19 infection.
\end{abstract}

\section{Introduction}

\section{Sars-Cov-2 infection}

The viral infection caused by the new Sars-Cov-2 (COVID-19), recorded its first cases in China at the end of 2019 , and within a few months it became a global pandemic. At the time of writing there were 4.71 million infected cases and 315,000 deaths with over 250 countries affected. (1) In most cases, COVID-19 infection is a mild disease, and resolves spontaneously with symptoms such as weakness, nausea, fever lines, while some people develop a severe infection characterized by a rapid decline in lung function and breathing difficulties. A minority of patients develop a generalized inflammatory state caused by a cytokinic storm responsible for multi-organ dysfunction, which can rapidly cause the death of the patient. To date, there are no direct antivirals and effective vaccines against Sars-Cov-2. (2)

\section{Diabetes patient}

Diabetes patients are more at risk than the general population of viral or bacterial infections. The immune system compromised by the metabolic pathology is characterized by a reduced response and effectiveness of neutrophil and T lymphocyte activity, and by the lack of an adequate humoral immune response, which make the patient with diabetes at high risk of viral or bacterial infection especially in the airways. (3) (4).

People with diabetes are therefore at increased risk of both COVID-19 infection and increased complications and mortality. General precautions to prevent COVID-19 infection are essential for people with diabetes. Patients with diabetes need continuous and regular blood glucose monitoring and optimal compliance with ongoing drug treatment, even in case of COVID-19 infection. For patients with severe COVID-19 diabetes, drugs such as glyptins, incretin mimetics or SGLT2 inhibitors can safely continue to be taken, and Ace 
inhibitors/ARB antihypertensives should also continue to be taken, unless contraindications or further evidence against their use develop. In particular, the diabetic patient has a higher risk of pneumonia mortality than the general population. In addition, during the SARS-Cov epidemic in 2003 similar to COVID-19, diabetes was identified as a risk factor for mortality from infection. Similarly, in another similar epidemic, such as MERS, the presence of diabetes was a risk factor for infection and serious complications. (5) (6). Similarly, among patients with influenza A (H1N1) in 2009, diabetes increased the risk of complications. (7).

\section{Sars-Cov-2 infection and diabetic patient}

In light of currently available data, people with diabetes appear to be more susceptible to Sars-CoV-2 infection. Predisposing risk factors are mainly secondary diseases caused by diabetes, such as coagulation dysfunction, inflammatory tissue status, nephropathy, cardiovascular diseases, immunodepressed status. An extensive epidemiological study of 72,314 patients with COVID-19 in China indicated that patients with diabetes have a mortality rate three times higher than all patients with COVID-19 (8). Diabetes is known to cause homeostasis dysfunction of the coagulation system and fibrinolytic cascade and hyperactive inflammation. These two components also manifest themselves in the more severe stages of Sars-Cov-2 infection. In the most severe stages of infection, the major complications are caused by a generalized hyperactive inflammatory state and coagulative dysfunction with risk of thrombosis and lung injury. In a study conducted on 174 patients with COVID-19 in Wuhan, China, people with diabetes had a higher concentration of inflammatory markers and a higher incidence of coagulopathy associated with higher mortality than those without diabetes (9). Another key aspect to highlight is that during the Sars-Cov epidemic it was noted that patients with viral infection had higher blood sugar levels than patients with infections caused by other viral agents. (10) Today it is known that Sars-Cov and Sars-Cov-2 use ACE-2 membrane glycoprotein to penetrate cells. In vitro and animal studies have shown that Sars-Cov binds ACE-2 also on pancreatic islands, damaging their function and causing a lack of adequate insulin production, (11) this biological mechanism is probably the cause of the hypeglycemia noticed in patients with Sars-Cov. Considering that sars-cov and Sars-Cov-2 share a high percentage of genomic similarity, and that Sars-Cov-2 also binds ACE-2, a risk of hyperglycemia could be hypothesized also in patients with COVID-19, as happened in the Sars-Cov epidemic. At the moment there are no data from epidemiological studies in this respect.

\section{Clinical pharmacological aspects in the management of the diabetic patient during the COVID- 19 pandemic}

\section{Antidiabetes agents}

The continuous control and monitoring of blood glucose is of fundamental importance for the diabetes patient, even more so during the COVID-19 pandemic. Patients with diabetes can safely continue to take routine drug therapies, to date there is no evidence that any anti-diabetes therapeutic agent can increase the risk of COVID-19 infection. Even if the patient with diabetes is affected by Sars-Cov-2, one should continue to monitor blood sugar levels and take the usual drug therapy. Probably in case Sars-Cov-2 infection leads the patient into serious complications, possible changes in dosage or medication could be considered, but should always be considered on a case-by-case basis. Several factors, including blood glucose, hemodynamic stability, nutritional status, kidney function, risk of hypoglycemia and drug interactions should be carefully assessed. However, the most commonly used antidiabetes medications in therapy may need to be modified or even discontinued in specific cases. Metformin is among the most commonly used medications for type 2 diabetes and is predominantly eliminated renal, in a COVID-19 patient careful monitoring of renal function should be performed to avoid risk of lactic acidosis caused by increased drug concentrations. Hypoxia may also increase the risk of lactic acidosis. (12) In addition some antivirals used to fight COVID-19 are inhibitors of the organic cation transporter OCT, metformin is a substrate of this transporter, inhibiting OCT may increase metformin concentrations in plasma, careful monitoring should be carried out to avoid this interaction. Some NSAIDs that can be used in the COVID-19 patient may decrease renal function and increase plasma metformin concentration. However, glucocorticoids may increase blood glucose, in which case a change in metformin dose may be considered, although at present the use of glucocorticoids does not seem to be recommended in the COVID-19 patient. Metformin may be discontinued in hospitalised and 
seriously ill patients due to the risk of lactic acidosis. Agonist glucagon peptide-1 receptor therapy (GLP1RA) should probably be temporarily discontinued in patients with hemodynamic instability (which impairs absorption from subcutaneous sites), renal dysfunction and gastrointestinal dysfunction. GLP-1RA may cause a slowdown in gastric emptying and may result in clinically significant reductions in the rate and degree of absorption of certain oral drugs administered simultaneously such as darunavir or remdesivir antivirals. (13) Common side effects of GLP-1RA include nausea, vomiting and diarrhoea, which in a COVID-19 patient with similar gastrointestinal symptoms may lead to electrolyte depletion, careful evaluation should be made, GLP-1RA are excreted predominantly renal, in a COVID-19 patient with renal dysfunction an increase in drug concentrations may occur. No particular interactions with anticoagulants such as LHMW or IL1 and 6 inhibitors are known.

Dipeptidil peptidase-4 (DPP4) inhibitors are associated with a low risk of hypoglycemia and are relatively safe in a wide range of renal functions, which is a strength in the COVID-19 patient. Although renal function is severely impaired, a dose modification of drugs in this class should be considered. (14) Another strength of these drugs is that there are no particular interactions with CYP inhibitors, and this is a very important aspect in a COVID-19 patient with diabetes, who is a complex patient and can use highly interacting drugs such as antivirals. Also, there are no particular interactions with anticoagulants. Probably the use of SGLT2 inhibitors is more complex in the COVID-19 patient, due to the risk of diabetic ketoacidosis, which can lead to potentially fatal cases. (15) The clinical condition of the patient, the concentration of ketone bodies, hyperglycemia, renal function are aspects to be taken into account for a possible suspension of treatment with SGLT-2. In addition, this category of drugs data suggest that the primary route of metabolism in humans is uridine 5'-diphosphorus glucuronidation (UDP) glucuronyltransferase (UGT) 1A3, 1A8, 1A9 and 2B7, and they are also OCT substrates. Interaction with antivirals such as darunavir and remdesivir should be carefully considered. There are no particular interactions with LHMW or IL-1 and 6 inhibitors. Insulin use should be carefully monitored due to the risk of hypoglycemia, and a dose change should be considered if the patient is on glucocorticoid therapy. In addition, a possible hypoglycemic additive effect due to hydroxychloroquine should be carefully evaluated. In patients with COVID-19 on sulfoniluree therapy, a change to insulin therapy should be considered because of the risk of high hypoglycemia in patients with Sars-Cov-2 infection. Caution with sulfonylureas should also be exercised in consideration of chloroquine, due to the risk of hypoglycaemia with both and if renal dysfunction is present. (16)

Thiazolidinediones such as pioglitazone should be carefully questioned for use in the COVID-19 patient, as this class of drugs is contraindicated in patients with hemodynamic instability, liver or cardiac dysfunction, which may be present in a severe COVID-19 infection Currently, existing evidence suggests that insulin is used compared to agents such as sulfonylureas or thiazolidinediones. (Figure 1) 


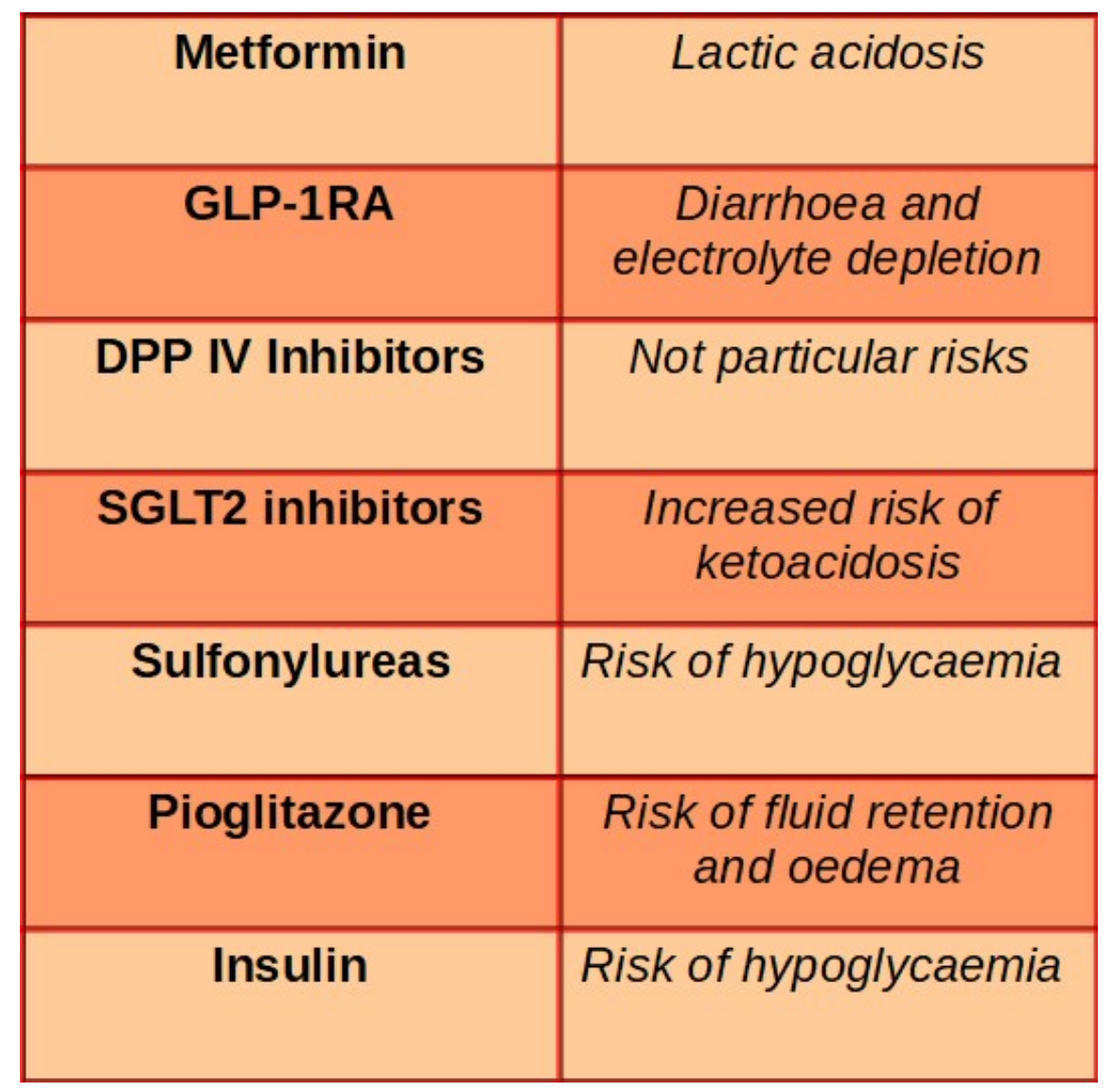

Figure 1: Main risks from antidiabetic agents in patients with COVID-19

Therapeutic agents for comorbidities

The therapy of the diabetic patient is complex and should aim not only at controlling glycemic homeostasis but also at a series of comorbidities that can worsen the clinical picture. The medical antihypertensive therapy of choice for the diabetic patient involves the use of ACE inhibitors or angiotensin antagonists2. These drugs not only reduce blood pressure and proteinuria but also slow the progression of diabetic nephropathy. To date there are conflicting and as yet inconclusive results on their use in this period of the COVID-19 pandemic, causing an increase in the risk of infection that they could cause by raising ACE-2 concentrations. It remains to be emphasized that there is currently no clear evidence for or against the use of ACEI/ARB in people with diabetes at risk or infected with Sars-CoV-2. (17). Currently, most international organisations recommend the continuation of ACEI/ARB therapy, unless there are explicit contraindications such as uncontrolled kalaemia or renal stenosis (18)(19). Patients with diabetes undergoing treatment with antiaggregants such as $100 \mathrm{mg}$ acetylsalicylic acid should continue to take them. There is currently no evidence of a possible suspension unless there are clear contraindications such as a risk of gastrointestinal bleeding or severe renal impairment. However, in severe COVID-19 patients co-administration of acetylsalicylic acid and corticosteroids or acetylsalicylic acid and heparin may lead to an increased risk of ulcer and gastrointestinal bleeding. In addition, there is currently no direct evidence for statin suspension in patients with diabetes and COVID-19. However, it is of great importance to consider that if the viral infection leads to renal damage (e.g. creatinine clearance $<60 \mathrm{ml} / \mathrm{min}$ ) or liver damage with increased transaminases, $(20-21)$ the statin concentration may increase causing a worsening of the clinical picture with risk of myopathy and liver damage. In addition, antiviral protease inhibitors such as lopinavir in association with statins should not be used because of drug interaction and the risk of increased statin concentrations (22). Therefore for the use 
of statins we suggest individualized decision on a case by case basis.

Anti-diabetes agent as potential therapeutic treatment for COVID-19

Some therapeutic agents used to treat diabetes have shown evidence of effectiveness against Sars-Cov and MERS, epidemics similar to Sars-Cov-2. It is now known that in the most severe stages of COVID-19 infection an overactive and uncontrolled inflammatory host system caused by a cytokinic cascade is responsible for multi-organ dysfunction and serious fatal lung lesions. The use of metformin has shown a decrease in inflammatory markers in patients with Sars-Cov and MERS, however its use in the severe COVID-19 patient should be carefully assessed in view of the risks described above. (23)(24) The use of DPP IV inhibitors (glyptine) is being studied in the COVID-19 patient. The DPP4 protein is expressed in many cells including alveolar epithelium and inflammatory cells. MERS-CoV uses DPP4 to enter host cells (25), but it is not known whether Sars-Cov-2 also uses the same protein to enter the cell, in addition to ACE-2. If this is demonstrated, the use of glyptins could decrease the risk of Sars-Cov-2 infection but this has not been demonstrated so far. The potential benefit in the treatment of Sars-Cov-2 infection with DPP IV inhibitors remains to be further investigated (26). Similar to metformin, the effects of reducing inflammatory markers are also known for GLP-1RA and have also demonstrated a potential therapeutic benefit in acute lung lesions (27). However, the available data are limited to experimental models and their benefit still remains to be studied.

\section{Conclusions}

The global pandemic from COVID-19 represents one of the greatest health challenges in human history. Pending the discovery of an effective vaccine and antivirals directed against Sars-Cov-2, it is essential to recognize factors that may increase the risk of infection or worsen complications from COVID-19. The identification of effective preventive and therapeutic strategies is urgently needed especially for patients with chronic diseases such as diabetes. Patients with diabetes are at greater risk in this pandemic period, and even diabetes therapy can be complex to manage during a COVID-19 infection. Clinical data and EBM to be translated into guidelines by scientific societies and countries are needed to better guide clinicians in the complex management of a diabetes patient who is COVID-19 positive.

\section{Main statements}

I, the undersigned, Francesco Ferrara and any other author, declare that:

- The manuscript was written entirely by the authors; - All authors made an equal contribution in the development of the paper; · We have no conflict of interest; · We have not received funding/source; · There are no sensitive data and no patients were recruited for this study; - The document does not conflict with ethical legislation.

Regards

The authors

\section{References}

1) World Health Organization HO (2020) Coronavirus disease 2019 (COVID-19) situationreport Available fromhttps://www.who.int/emergencies/diseases/novel-coronavirus-2019/situation-reports.

2) Lin L et al. (2020) Hypothesis for potential pathogenesis of Sars-CoV-2 infection a review of immune changes in patients with viral pneumonia. Emerg Microbes Infect 9(1):727- 732 .

3). Carey IM et al. (2018) Risk of infection in type1 and type 2 diabetes compared with the general population: a matched cohort study. Diabetes Care 41(3):513-521.

4). Geerlings SE, Hoepelman AI (1999) Immune dysfunction in patients with diabetes mellitus (DM). FEMS Immunol Med Microbiol 26(3-4):259-265. 
5). Yang YM et al (2017) Impact of comorbidity on fatality rate of patients with Middle East respiratory syndrome. Sci Rep 7(1):11307.

6). Garbati MA et al (2016) A comparative study of clinical presentation and risk factors for adverse outcome in patients hospitalised with acute respiratory disease due to MERS coronavirus or other causes. PLoS One 11(11):e0165978.

7) Allard R et al. (2010) Diabetes and the severity of pandemic influenza A (H1N1) infection. Diabetes Care 33(7):1491-1493.

8) Wu Z, McGoogan JM (2020) Characteristics of and important lessons from the coronavirus disease 2019 (COVID-19) outbreak in China: summary of a report of 72314 cases from the Chinese Center for Disease Control and Prevention. JAMA. 323(13):1239.

9) Guo W et al (2020) Diabetes is a risk factor for the progression and prognosis of COVID-19. Diabetes Metab Res Rev e3319.

10) Yang JK et al (2006) Plasma glucose levels and diabetes are independent predictors for mortality and morbidity in patients with SARS.DiabetMed23(6):623-628.

11) Yang JK et al. (2009) Bindingof SARS coronavirus to its receptor damages islets and causes acute diabetes. Acta Diabetol 47(3):193-199.

12) RalphDeFronzo et al. (2016) Metformin-associated lactic acidosis: Current perspectives on causes and risk Metabolism Volume 65, Issue 2, February 2016, Pages 20-29

13) Liu, J et al. (2017). "Incretin based treatments and mortality in patients with type 2 diabetes: systematic review and meta-analysis". BMJ (Clinical Research Ed.). 357: j2499.

14) McIntosh, C et al. (2005). "Dipeptidyl peptidase IV inhibitors: How do they work as new antidiabetic agents?". Regulatory Peptides. 128 (2): 159-65.

15) Bonora BM et al. (2020). "Extraglycemic Effects of SGLT2 Inhibitors: A Review of the Evidence". Diabetes, Metabolic Syndrome and Obesity: Targets and Therapy. 13: 161-174.

16) Hemmingsen B (2014). "Sulfonylurea versus metformin monotherapy in patients with type 2 diabetes: a Cochrane systematic review and meta-analysis of randomized clinical trials and trial sequential analysis". CMAJ Open. 2 (3): E162-75.

17) Fang L et al. (2020) Are patients with Hypertension and diabetes mellitus at increase drisk forC OVID-19 infection? Lancet Respir Med 8(4):e21.

18) Vaduganathan $\mathrm{M}$ et al. (2020) Renin-angiotensin-aldosterone system inhibitors in patients with Covid19. N Engl J Med 382(17):1653- 1659.

19) Patel AB, Verma A (2020) COVID-19 and angiotensin-converting enzyme inhibitors and angiotensin receptor blockers: what is the evidence? JAMA (3): E162-75.

20) Driggin E et al. (2020) Cardiovascular considerationsfor patients, health care workers, andhealth systems during the coronavirus disease 2019 (COVID-19) pandemic. J Am Coll Cardiol. https://doi.org/10.1016/j.jacc.2020.03.031 63.

21) Li Y, Wang M et al. (2020) Acute cerebrovascular disease following COVID-19: a single center, retrospective, observational study. SSRN. https://doi.org/10.2139/ssrn.3550025 64 .

22) Bangash MN et al. (2020) COVID-19 and the liver: little cause for concern. Lancet Gastroenterol Hepatol 11(1):2.

23) Zumla A et al. (2020) Reducing mortality from 2019-nCoV: host-directed therapies should be an option. Lancet 395(10224):e35-e36. 
24) Zumla A et al. (2016) Coronaviruses - drug discovery and therapeutic options. Nat Rev Drug Discov 2016; 15: 327-47.

25) Raj VS et al (2013) Dipeptidyl peptidase 4 is a functional receptor for the emerging human coronavirusEMC. Nature 495(7440):251-256.

26) Iacobellis G (2020) COVID-19 and diabetes: can DPP4 inhibition playarole?DiabetesResClinPract162:108125.

27) Feng Y et al (2020) Effect of hCMSCs and liraglutide combination in ALI through cAMP/PKAc/ $\beta$ catenin signaling pathway. Stem Cell Res Ther 11(1):2. 